\title{
Reflets
}

Revue ontaroise d'intervention sociale et communautaire

\section{Les jeunes et leurs visions du féminisme}

\section{Christiane Bernier et Renée C. Mallet}

Volume 3, numéro 2, automne 1997

Visibles et Partenaires : Pratiques et recherches féministes

URI : https://id.erudit.org/iderudit/026176ar

DOI : https://doi.org/10.7202/026176ar

Aller au sommaire du numéro

Éditeur(s)

Reflets : Revue ontaroise d'intervention sociale et communautaire

ISSN

1203-4576 (imprimé)

1712-8498 (numérique)

Découvrir la revue

Citer cet article

Bernier, C. \& Mallet, R. C. (1997). Les jeunes et leurs visions du féminisme. Reflets, 3(2), 128-142. https://doi.org/10.7202/026176ar

\section{Résumé de l'article}

Nous présentons, dans cet article, une partie des résultats d'une enquête qui portait sur les opinions des jeunes, particulièrement des jeunes femmes, sur le féminisme. Cette enquête a été effectuée auprès d'étudiantes et d'étudiants inscrits dans divers programmes de baccalauréat. Les résultats présentés ici démontrent que les jeunes femmes d'aujourd'hui donnent, dans l'ensemble, une définition relativement favorable du féminisme, mais ne semblent pas voir comme nécessaire leur implication dans le mouvement.
Tous droits réservés (C) Reflets : Revue ontaroise d'intervention sociale et communautaire, 1997
Ce document est protégé par la loi sur le droit d'auteur. L'utilisation des services d'Érudit (y compris la reproduction) est assujettie à sa politique d'utilisation que vous pouvez consulter en ligne.

https://apropos.erudit.org/fr/usagers/politique-dutilisation/ 


\section{Les jeunes et leurs visions du féminisme}

Nous présentons, dans cet article, une partie des résultats d'une enquête qui portait sur les opinions des jeunes, particulièrement des jeunes femmes, sur le féminisme. Cette enquête a été effectuée auprès d'étudiantes et d'étudiants inscrits dans divers programmes de baccalauréat. Les résultats présentés ici démontrent que les jeunes femmes d'aujourd'hui donnent, dans l'ensemble, une définition relativement favorable du féminisme, mais ne semblent pas voir comme nécessaire leur implication dans le mouvement.

\section{Christiane Bernier}

Professeure, Département de sociologie, Université Laurentienne

\section{Renée C. Mallet}

Étudiante, École de service social, Université Laurentienne

\section{Introduction}

Est-il vrai que les jeunes d'aujourd'hui ont une telle vision du féminisme que cela les détourne de militer activement au sein d'organismes qui promeuvent la transformation des espaces symboliques féminin et masculin? Est-il possible que l'appellation même de «féministe» soit encore si connotée et renvoie à une telle image de repoussoir que les jeunes femmes ne veulent surtout pas y être identifiées? Et si tel est le cas, comment ces jeunes femmes se définissent-elles? Quels sont les éléments à partir desquels elles construisent leur identité de femme, dans un monde où la lutte des femmes pour l'espace social et économique n'est certes pas gagnée? 
Pour le savoir, Renée, avec la collaboration de Christiane, a voulu mener une recherche auprès de jeunes adultes universitaires de niveau baccalauréat, dans le cadre d'un cours portant sur les rapports de sexe. L'objectif de la recherche ne prétendait pas dévoiler dans quelle mesure les jeunes sont informé-es ou non des diverses politiques qui sont favorables aux femmes dans la société contemporaine, ni même de mesurer leur savoir sur les diverses tendances du féminisme actuel et les débats multiples qui s'y expriment. Il s'agissait plutôt de découvrir à quoi la symbolique féministe correspond dans leur esprit.

Ce sont les résultats partiels de cette enquête qui sont livrés ici, agrémentés des réflexions qu'ont suscité l'analyse des résultats chez chacune des auteures.

\section{Contexte de la recherche}

«...l'expérience de Renée l'a amenée à penser que les personnes de moins de trente ans, et particulièrement les jeunes hommes, ont une opinion assez négative du féminisme...»
Militante féministe dans des organismes sociaux et parauniversitaires, l'expérience de Renée l'a amenée à penser que les personnes de moins de trente ans, et particulièrement les jeunes hommes, ont une opinion assez négative du féminisme, alors que les jeunes femmes ne sentent pas la nécessité de travailler pour la "cause des femmes». Comment alors les «recruter» pour venir grossir les rangs devenus trop maigres des femmes actives dans le mouvement?

Christiane s'intéresse plutôt à un autre aspect de la question : si les convictions de Renée s'avèrent, où s'en va donc le féminisme? Le changement de génération en sonnera-t-il le glas? Selon elle, ce qui importe ce n'est pas tant que le Féminisme avec un grand $F$ doive être maintenu dans sa version idyllique, mais de découvrir si son «détournement» ou son «évitement» n'entraîne pas un retour en arrière sur des valeurs dites «féminines» présentées comme vertu.

Bien sûr, la question de la relève, au sein du mouvement féministe, n'est pas nouvelle. Déjà, à la fin des années 1980, les féministes de la première heure, épuisées et inquiètes sans doute, 
"...le féminisme, quelle qu'en soit la tendance théorique, pourrait ne pas

répondre à leurs besoins de jeunes femmes d'aujourd'hui...» se demandaient bien comment - et surtout à qui - passer le flambeau. Non résolue à cette époque, cette question demeure particulièrement épineuse pour diverses raisons.

Une des raisons majeures réside assurément dans le contexte dans lequel vivent les jeunes femmes d'aujourd'hui, qui ont bénéficié, à double titre, du travail de leurs mères féministes. D'une part, elles n'ont plus à revendiquer ce qui a été acquis et qui est devenu monnaie courante, non seulement dans le langage quotidien, mais aussi dans les représentations sociales (le principe de l'égalité constitutionnelle des sexes, le droit de la femme non accompagnée aux espaces publics, l'autonomie juridique de la femme mariée, l'accès au patrimoine après divorce, le partage de la responsabilité de la garde des enfants, les lois anti-viol, l'antiharcèlement...). D'autre part, après voir été témoins du coût physique et psychologique qu'ont dû payer les superwomen de leur enfance pour tenter de tout faire, tout concilier, tout changer, elles en tirent peut-être une leçon d'expérience, en se disant «à moi, ça n'arrivera pas!», et se refusent à tout militantisme.

Mais ces raisons, que l'on invoque à la décharge des jeunes femmes d'aujourd'hui qui, en grande majorité, ne se sentent pas concernées par le mouvement féministe, sont-elles les bonnes? Serait-ce ce qu'elles répondraient si la question leur était posée? Ou bien ont-elles une vision du féminisme ou des critiques portant sur d'autres éléments, d'autres enjeux? Par exemple, le féminisme, quelle qu'en soit la tendance théorique, pourrait ne pas répondre à leurs besoins de jeunes femmes d'aujourd'hui, comme l'ont mentionné certaines d'entre elles (Godin 1990; Michaud 1994; Vanstone 1996)? Ou que les rapports hommes/femmes instigués par les revendications féministes ont impliqué trop d'agressivité et qu'elles aimeraient bien en faire changer la teneur...

\section{L'enquête}

L'enquête a été menée au mois de mars 1997, dans les classes de l'Université Laurentienne de Sudbury, sur une population d'étudiantes et d'étudiants de niveau baccalauréat, toutes disciplines confondues. 


\section{La collecte de données}

La collecte de données a été réalisée à partir d'un questionnaire auto-administré, comprenant, outre les questions d'ordre sociodémographique de base :

1. Une échelle de Likert, à partir de laquelle on demandait aux répondantes et aux répondants de donner leur opinion sur le féminisme en se prononçant sur une douzaine d'énoncés ${ }^{1}$.

2. Une question ouverte portant sur leur définition du féminisme (Qu'est-ce que le féminisme pour toi?) dont l'objectif était d'identifier à partir de quels mots, de quels éléments les jeunes parlent du féminisme; cela explique pourquoi nous avons tenu à rester résolument vagues sur le mot comme tel de féminisme, parce qu'en avoir identifié des tendances aurait, à notre sens, biaisé les résultats.

\section{L'échantillon}

L'échantillon retenu, comme l'indique le tableau 1, comprend 164 personnes inscrites dans divers programmes de baccalauréat, de la première à la quatrième année. Le groupe visé par l'enquête était les jeunes femmes de moins de trente ans; cependant, aux fins d'exemples et de comparaison, nous avons conservé les données provenant des autres répondants. Deux sous-échantillons ont ainsi été constitués: celui des personnes de sexe masculin et celui des personnes de plus de trente ans, recrutées dans les mêmes classes. On remarquera sans peine le déséquilibre entre les sousgroupes de l'échantillon.

Tableau 1 - Profil de l'échantillon global $(n=164)$

\begin{tabular}{ll|lr|lr}
\hline \multicolumn{2}{c|}{ Sexe } & \multicolumn{2}{c|}{ Âge } & \multicolumn{2}{c}{ Programme } \\
\hline Féminin & $=140$ & 19 ans & 55 & Sciences sociales & 55 \\
Masculin & $\mathrm{n}=23$ & 20 ans & 36 & Humanités & 15 \\
Manquant & $\mathrm{n}=1$ & 21 ans & 26 & Écoles professionnelles & 74 \\
& & 22 à 29 ans & 35 & Sciences & 18 \\
& & 30 et plus & 12 & Manquant & 2 \\
\hline
\end{tabular}




\section{L'analyse des résultats}

Les données obtenues ont été soumises à deux types d'analyse: quantitative et qualitative.

En premier lieu, les réponses à l'échelle d'attitude ont été traitées à l'aide de différents tests statistiques (corrélations, tests $\mathrm{t}$ et régressions multiples). Nous voulions, à partir de ces tests, obtenir deux sortes d'informations. D'une part, identifier l'influence de l'âge et du sexe sur la variation des opinions exprimées pour chaque énoncé. D'autre part, nous avons cherché à voir si des tendances réelles, en faveur ou contre le féminisme - toujours en fonction de l'âge et du sexe - , pouvaient être identifiées dans l'ensemble des réponses. En effet, les énoncés avaient été construits de telle sorte que des regroupements étaient possibles, identifiant certaines valeurs à l'égard du féminisme: soit en faveur, soit à l'encontre, voire encore sans connotation particulière lorsque l'énoncé est de type descriptif ou n'implique pas une position claire. Cette axiologisation volontaire de l'ensemble des énoncés avait été conçue pour identifier trois blocs d'attitudes: proféministes, anti-féministes et indéterminées.Mais, pour des raisons liées au type d'échantillon obtenu (manque d'homogénéité entre les sous-groupes, déséquilibre des échantillons contrôles), les résultats de cette partie de l'enquête, même s'ils ont été soumis aux traitements statistiques appropriés, ne peuvent être retenus sans risque. Aussi la prudence nous impose-t-elle de les soumettre à une vérification ultérieure: ils feront donc l'objet d'une recherche subséquente.

En deuxième lieu, les données provenant des définitions du féminisme ont été traitées à partir d'une analyse de contenu de type thématique; nous avons donc identifié les catégories et les sous-catégories auxquelles renvoient les éléments mentionnés dans les énoncés de chacune des définitions.

Nous présentons ici les résultats de l'analyse qualitative. 


\section{Lecture des résultats : les éléments d'une représentation sociale}

À la question "Qu'est-ce que le féminisme pour toi?», un total de 146 définitions ont été retenues parmi les réponses obtenues : dix personnes s'étant abstenues et huit autres ayant déclaré «je ne sais pas». Ces définitions, relativement succinctes en général, sont assez disparates du point de vue de la forme : elles peuvent se présenter comme un ensemble d'énoncés articulés, émettant plusieurs idées, ou comme une vision réfléchie du phénomène...

Pour moi le féminisme c'est l'étude de la condition des fermmes. C'est aussi l'affirmation de nos droits et liberté [s] en tant que femme (F156, 20 ans).

...tantôt elles ne seront composées que de quelques mots: un mode de vie! (F154, 21 ans).

Mais, dans l'ensemble, les thèmes abordés sont récurrents et la représentation que l'on y développe du féminisme est, somme toute, assez positive : trente définitions seulement seront en partie ou totalement négatives, comme l'indique le tableau 2.

Pour obtenir les catégories d'analyse, nous avons relevé tous les thèmes abordés dans chaque définition. Lorsqu'il y avait plus d'un thème renvoyant à la même catégorie, nous inscrivions la définition dans une sous-catégorie comprenant les deux thèmes: ce pourquoi il semble y avoir, dans le tableau 2, quelques répétitions, qui, de fait, n'en sont pas. Par ailleurs, lorsque la définition comprenait plusieurs thèmes différents ou qu'elle contenait une pensée plus complexe, elle a été catégorisée dans la section Définitions complexes; c'est pourquoi cette catégorie n'a pas de sous-thèmes. En ce qui concerne le deuxième groupe de

«...il nous paraissaît intéressant de voir dans quelle mesure le féminisme était perçu comme un ensemble, un mouvement...» définitions, Un mouvement ou groupe [s] en faveur de l'égalité, il a fait l'objet d'une hypothèse particulière; en effet il nous paraissait intéressant de voir dans quelle mesure le féminisme était perçu comme un ensemble, un mouvement, une organisation, plutôt qu'uniquement comme des actions accomplies par des acteures individuelles. 
Nous comptons plus ou moins le tiers des «définitions» qui en sont réellement, c'est-à-dire qui découlent d'une réflexion articulée et qui, d'un point de vue féministe, sont intéressantes. Dans ces définitions, contrairement à l'ensemble des autres énoncés, on fait plus que présenter uniquement l'objectif que l'on croit être celui du féminisme (l'égalité entre les sexes): on élabore, sur sa dimension historique, sur sa nécessité actuelle ou future...

Dans l'ensemble cependant, on définit le féminisme moins comme organisation ou ensemble de groupes de femmes que comme mouvement et on présente, comme équivalent, le fait de se battre pour l'égalité de la femme avec l'homme et pour l'égalité entre les sexes ou les droits égaux, sans comprendre que cette vision donne encore à l'image de l'homme le statut de référent dans les représentations des aspirations.

La plupart des autres définitions sont plus ou moins des opinions spontanées qui renvoient à la rumeur sociale. Quelques exemples d'énoncés, pour chacune des catégories, suffiront à rendre le propos.

\section{Tableau 2 - Liste et fréquence des thématiques abordées dans les définitions du féminisme $(n=146)$}

\begin{tabular}{|c|c|c|}
\hline Catégories thématiques & $\mathbf{F}$ & Sous-thèmes \\
\hline Définitions complexes & 20 & \\
\hline $\begin{array}{l}\text { Mouvement/groupe-s en } \\
\text { faveur de l'égalité }\end{array}$ & 20 & \\
\hline $\begin{array}{l}\text { Définitions autour du thème } \\
\text { des luttes }\end{array}$ & 25 & 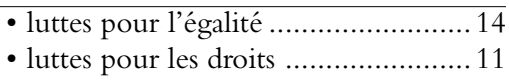 \\
\hline $\begin{array}{l}\text { Définitions autour des } \\
\text { thèmes égalité et droits }\end{array}$ & 31 & 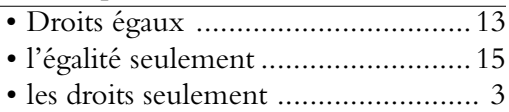 \\
\hline $\begin{array}{l}\text { Définition autour de thèmes } \\
\text { diversifiés }\end{array}$ & 20 & $\begin{array}{l}\text { - } \text { autonomie/ } \\
\text { indépendance de la femme ............ } 6 \\
\text { - autour du pouvoir ...................... } 3 \\
\text { - discrimination contre les femmes ..... } 4\end{array}$ \\
\hline $\begin{array}{l}\text { Définitions ambivalentes: } \\
\text { l'exagération du féminisme }\end{array}$ & 15 & $\begin{array}{l}- \text { Bon...mais .................................. } 8 \\
\text { - mouvement trop extrême ............. } 7\end{array}$ \\
\hline Définitions négatives & 15 & $\begin{array}{l}\text { - femmes qui se sentent supérieures ... } 7 \\
\text { - discrimination envers les hommes .... } 3 \\
\text { - propos sexistes .......................... } 5\end{array}$ \\
\hline
\end{tabular}


"...il y a plusieurs groupes féministes, car les femmes n'ont pas encore gagné tout le respect voulu dans les carrières, en plus...»
- Définitions complexes

- Lorsque les femmes ont gagné plusieurs droits : vote, emploi. Son début est pendant la $2^{\mathrm{e}}$ G-M. Même encore aujourd'hui, il y a plusieurs groupes féministes, car les femmes n'ont pas encore gagné tout le respect voulu dans les carrières, en plus des stéréotypes (F34, 19 ans) ${ }^{2}$.

- Mouvement qui vise à améliorer la situation des femmes dans la société. Vise à obtenir une certaine égalité entre les deux sexes. Permet aux femmes de s'exprimer et de dénoncer qu'elles n'apprécient pas (F49, 20 ans).

- Le féminisme signifie le statut que porte la femme dans son milieu communautaire, social, culturel et économique. Le féminisme signifie aussi les actions entreprises par les gens pour apporter des meilleures conditions de vie par rapport au statut de la femme (F96, 23 ans).

- Le féminisme c'est une pensée d'égalité entre les hommes et les femmes, les hommes peuvent être féministe [s]. La pensée qu'il n'y a pas de sexe [plus supérieur qu]'un autre (F106,20 ans).

\section{- Mouvement ou groupe(s) en faveur de l'égalité}

- Le féminisme est un mouvement qui réclame des droits égalitaires entre le sexe féminin et masculin (F56, 19 ans).

"Mouvement prônant les droits des femmes sur le marché du travail...»
- Mouvement prônant les droits des femmes sur le marché du travail, le domaine juridique. Prône l'équité sexuelle (H31, 21 ans).

- Le féminisme est un mouvement dans lequel les femmes veulent l'égalité de sexe. C'est-à-dire même paie, même traitement au travail de même que égalité partout. (F18, 20 ans).

- Un «mouvement», un [e] attitude qui reflète le pouvoir et la détermination de femmes intelligentes et actives (aware) (F145, 20 ans).

\section{- Autour du thème des luttes}

- Le féminisme est plutôt quelque chose, idéologie, pour lutter pour l'égalité (F11, 21 ans). 
"La lutte pour

l'égalité..."
"C'est le fait qu'une femme est considérée comme moins importante qu'un homme...»
- Des gens qui se battent pour que la femme soit sur le même pied d'égalité que les hommes. Pour qu'il n [i] [est] ait pas de discrimination (F14, 21 ans).

- Pour moi le féminisme est simplement que les femmes ont les mêmes droits que les hommes. En d'autre [s] mot [s] le féminisme lutte pour qu'il y ait de l'égalité entre les deux sexes (H06, 21 ans).

- La lutte pour l'égalité entre les sexes, la fierté de son genre. La lutte pour éliminer le sexisme (F74, 21 ans).

\section{- Autour des thèmes: égalité et droits}

- La libération des droits de la femme qui ont longtemps été considéré [s] comme inférieur [s] à ceux de l'homme (H117, 21 ans).

- C'est le droit de la femme d'avoir sa place au sein de la société. C'est l'égalité. C'est le point de vue de la femme dans diverses situations familiales, sociales, politiques, etc.(F01, 22ans).

- Le féminisme est pour moi les gens qui croi [en] $t$ aux droits des femmes. Qu'elles devraient avoir les mêmes privilèges que les hommes (F118, 19 ans).

- D'être fière d'être femme. Que je suis égale à la population (femme et homme). J'ai le droit de faire tout ce que l'homme fait si je le désire (égalité) (F97, 23ans).

\section{- Autour de thèmes diversifiés}

- Une femme qui prend toutes décisions face à son corps et son entière personne (F12, 32 ans).

- C'est d'être une femme indépendante, d'avoir son propre succès, se surpasser en soi et être confiante, ne pas se soumettre à aucune idéologie sociale (F59, 19 ans).

- C'est le fait qu'une femme est considérée comme moins importante qu'un homme dans tous les aspects. Ex: au travail, à l'école, dans la société (F46, 19 ans).

- Les femmes qui essaient d'affermir leur pouvoir dans un monde surtout dominé par le sexe masculin (F128, 19 ans). 
- Définitions ambivalentes

- Il me semble qu'il ne faut pas qu'on utilise «féminisme» encore parce que ça donne des idées des femmes enragées qui veulent être vues comme supérieures. C'est supposé que les femmes se battent pour les “equal opportunities". C'est à dire qu'il ne faut pas avoir le même nombre de femmes que [d'] hommes à une institution, mais que les femmes ont vraiment l'occasion égal [e] d'être acceptées ou employées (F123, 19 ans).

- C'est qu'une femme se pense meilleur [e], supérieur [e], elle [s] sont éga [ux] dans tous les sens et elle travaille pour l'égalité (F116, 19 ans).

- Ce sont des femmes qui désirent promouvoir les droits des femmes. C'est un groupe à mon avis qui pousse un peu trop fort et qui mène à l'exagération (F80, 20 ans).

- Ce sont des gens qui poussent pour l'égalité de la femme par rapport aux hommes. Par contre, il y en a qui pousse [nt] l'affaire beaucoup trop loin, exagère [nt] leur point de vue (F105, 19 ans).

- Pour moi le féminisme est quelqu'un qui utilise la position de la femme d'aujourd'hui à un cas extrême. Elles veulent lutter contre les hommes au lieu de travailler avec eux afin d'améliorer leur situation (F16, 20 ans).

«Le féminisme pour _ Le féminisme pour moi est un mouvement extrême à atteindre, moi est un mouvement extrême...» un objectif non-atteignable en réalité (F58, 19 ans).

\section{- Définitions négatives ou sexistes}

- C'est lorsque les femmes croient qu' elles sont meilleures que les hommes, que les hommes sont bon [s] à rien (H120, 19 ans).

- Femme qui pense qu'elle est supérieure aux hommes. Contredit tou [t] sur les hommes (H143, 19 ans).

- Un mouvement avec des points forts ainsi que faibles. Il aide parfois aux problèmes de femmes, mais en majorité elles ne font que descendre les hommes (F72, 21 ans).

"C'est être sexiste..." - C'est être sexiste; contre plusieurs choses concernant les mâles (F77, 22 ans). 
- Quelque chose de fatiguant! Les féministes poussent trop les choses à des sujets extrêmes! Le féminisme c'est du lavage de cerveau (F39, 20 ans).

- le support extrême (presque "right-wing”) des droits féminins (H139, 19 ans).

- des femmes [qui pensent] que les féministes sont la race supérieure et qu'elles n'ont pas de besoin de l'homme sauf pour reproduction et ouvrir des pots de conserve (H152, 20 ans).

Il ne fait aucun doute que les thèmes de l'égalité et des droits sont ceux autour desquels tournent la majorité des définitions (76/146). Et c'est justement en cela que réside la principale différence, dans les représentations du féminisme, entre les jeunes femmes actuelles et celles des années 1970 : alors que les féministes d'antan auraient parlé d'aliénation, de revendication, d'oppression des femmes, de victimisation, de patriarcat, de libération même, ici,les maîtres-mots sont égalité et droits ${ }^{3}$. Cela seul pourrait suffire à nous convaincre que, au delà de tous les courants, de toutes les factions, de toutes les tendances, le féminisme libéral a remporté tous les suffrages et que c'est celui qui, non seulement passera à l'histoire, mais surtout qui est le seul que célèbrent les jeunes femmes et certains des jeunes hommes des années 1990, lorsque les unes et les autres parlent positivement du féminisme.

Parce que, mis à part les quelques commentaires négatifs -

"...le féminisme est généralement présenté de façon positive...»

"...la majorité des jeunes fermmes d'aujourd'hui ne se sentent pas contraintes...» qui sont loin de faire l'unanimité — le féminisme est généralement présenté de façon positive, et l'on voit bien qu'il fait partie des représentations sociales des répondants, au même titre, pourraiton dire, que d'autres éléments symboliques construisant leur vie : la nécessité de faire des études, la difficulté de se trouver un bon emploi, l'importance d'avoir une reconnaissance sociale, etc.

Le deuxième élément que l'analyse de ces résultats souligne, c'est qu'il apparaît assez clairement que la majorité des jeunes femmes d'aujourd'hui ne se sentent pas contraintes, ne se voient pas opprimées ou victimes de l'idéologie patriarcale; elles vivent avec l'idée que la discrimination contre les femmes relève d'une question d'aménagements ponctuels de certains secteurs, particulièrement au niveau du marché du travail, et avec l'assurance 
qu'une fois les quelques luttes nécessaires menées, elles obtiendront l'égalité sexuelle réelle. C'est une illusion bien propre au féminisme libéral!

Finalement, on voit bien aussi que leurs principales critiques portent sur l'attitude des femmes des années 1970: elles condamnent le féminisme de ces années-là pour ne pas avoir intégré les hommes au processus de transformation de la situation des femmes et les féministes d'antan pour avoir été trop radicales dans leurs revendications et leur détermination. On ne s'étonne pas, dès lors, que le partage des tâches domestiques, la lutte contre la pornographie, la lutte pour le droit à l'avortement libre et gratuit, ne fassent plus partie du discours et ne soient jamais mentionnés comme éléments essentiels de la revendication des femmes pour atteindre l'égalité.

On trouve donc là, en partie, confirmation aux hypothèses soulevées au début de la recherche.

\section{Conclusion}

"...le féminisme... est passé dans la coutume sociale.»
Les résultats de l'enquête sur la vision du féminisme par les jeunes universitaires amènent, certes, certains constats, mais imposent aussi certaines réflexions. Particulièrement à travers l'analyse des définitions, on a pu voir que des tendances pro-féministes se manifestent très clairement. Mais la vision du féminisme qui y est présentée nous laisse perplexe. L'analyse de ces définitions qui nous montrent sans cesse les mêmes opinions, les mêmes jugements, laisse apparaitre l'effet répété d'un discours devenu social et passé dans les représentations. Le dire sur le féminisme ne tient plus de la marginalité, de l'exclusion, de la pensée révolutionnaire. Il est passé dans la coutume sociale.Voilà pourquoi, peut-être, on obtient tant de définitions répétitives, mais si peu de réflexions plus complexes: pour penser le différent, il faut penser de façon critique à partir de ce qui est, et non seulement s'en faire l'amplificateur... 
"On ne pense pas le féminisme, chez les jeunes femmes d'aujourd'hui, on vit avec son existence et l'obligation d'avoir une position claire à son sujet...»
La distinction entre pensée articulée et redite sociale apparât clairement aussi dans les différents glissements sémantiques qui, dans les définitions, font passer le discours sur l'objet de réflexion (le féminisme) à un discours sur les sujets ou les acteures qui le personnifient dans les représentations de la répondante ou du répondant (le féminisme, c'est des femmes qui...); et ce saut discursif advient d'autant plus facilement que l'énoncé produit sera de premier niveau, de l'ordre du préjugé, une opinion toute faite, un jugement négatif.

Tout se passe comme si le féminisme n'était pas un réel sujet de réflexion, pour les jeunes femmes, mais, au contraire, un sujet de réaction: l'important étant soit de s'en démarquer, soit d'en atténuer la portée de la représentation dans sa vie sociale, comme si cela était pour porter atteinte à leur image d'elles-mêmes, à leurs relations sociales, à leur carrière, à leur futur. On ne pense pas le féminisme, chez les jeunes femmes d'aujourd'hui, on vit avec son existence et l'obligation d'avoir une position claire à son sujet, pour ne pas être étiquetée. Peu s'interrogent sur la pertinence de l'étiquette, pourtant. Comment alors, avec une telle vision du féminisme, peut-on avoir envie de s'y impliquer, peut-on voir la nécessité de son action?

Cet état de fait est assurément le meilleur indicateur de la réussite du féminisme des années 1970, tant décrié. Mais il porte le poids de ses contradictions: il aurait été moins radical que l'étiquette sociale en ferait moins peur aux jeunes femmes d'aujourd'hui, mais elles auraient moins d'espace symbolique pour le clamer. On ne sort pas d'un tel paradoxe.

Pourtant, au delà de ces constations, il est quelque part rassurant de voir que les jeunes femmes actuelles, ne sont non seulement pas totalement dupes de la nouvelle position sociale des femmes, mais, qu'en plus, elles affirment devoir asseoir leur propre réussite, leur propre pouvoir en se faisant valoir à la fois comme femme et comme individu dans un monde qu' elle reconnaisse encore, malgré tout, masculin à plusieurs égards.

Cette certitude inaliénable de leurs droits et le regard, somme toute positif, qu'elles portent sur le féminisme - lorsqu'elles ne se sentent pas contraintes de s'en démarquer dans leur vie 
personnelle - nous font voir qu'il y a bien là un processus en marche. Ils sont les espoirs sinon les garants de l'avenir au féminin, en tous les cas, les bastions de sa construction au présent.

\section{Bibliographie}

BERNIER, Christiane (1991). Libéralisme et Féminisme: une lecture épistémologique, Thèse de Ph.D. sociologie, Université de Toulouse le Mirail, non publiée.

GODIN, Colette (1990). "Que pensent Danièle et ses copines: le mouvement féministe et la relève», Femmes d'action, vol. 19, no 3, 19.

GUINDON, Geneviève (1996). Les opinions et perceptions de jeunes femmes à l'égard du féminisme, Thèse de M.A., Université du Québec à Montréal, non publiée.

MICHAUD, Lyne (1994). «Moi, une féministe», Femmes d'action. vol. 23, no 4, 33-35.

MOLLER OKIN, Susan (1995). «Sur la question des différences», dans La Place des femmes: les enjeux de l'identité et de l'égalité au regard des sciences sociales, Actes du colloque de préparation française à

la 4 e conférence mondiale sur les Femmes, Paris, Éditions La Découverte, 57-69.

PICQ, Françoise (1993). Libération des femmes. Les années-mouvement, Paris, Seuil.

PICQ, Françoise (1997). «Un homme sur deux est une femme:les féministes entre égalité et parité», Les Temps Modernes, no 593, 219-237.

VANSTONE, Susan (1996). «Young Women and Feminism in Northern Ontario», dans M. Kechnie et M. Reitsma-Street (éd.), Changing Lives:Women in Northern Ontario, Toronto, Dundurn Press, 325-334.

\section{Notes}

1. Ces énoncés se voulaient une synthèse des idées les plus véhiculées par les jeunes femmes lorsqu'elles s'expriment sur le féminisme. Pour exprimer leur opinion, les répondants et les répondantes devaient encercler une valeur, pour chaque énoncé, sur une échelle de Likert à 6 niveaux, où $1=$ pas du tout d'accord et $6=$ tout à fait d'accord. Il s'agit des énoncés suivants:

1) Le féminisme n'a pas d'importance pour moi;

2) Le féminisme est encore nécessaire;

3) Le féminisme est une affaire de femmes;

4) Il y a des féministes dans mon entourage;

5) Le féminisme ne répond pas aux besoins des jeunes femmes d'aujourd'hui;

6) Je ne m'identifie pas comme jeune féministe;

7) Le féminisme m'aide dans ma vie personnelle;

8) Je ne suis pas féministe mais je crois à l'égalité de la femme;

9) J'aurais peur de me présenter comme féministe;

10) Le féminisme défend des dossiers importants pour moi; 
11) Le féminisme, ça concerne autant les hommes que les femmes;

12) Je n'aimerais pas que mon entourage me voie comme féministe.

2. Les énoncés présentés proviennent tous des définitions prises sur les questionnaires. Les informations entre parenthèses renvoient au sexe de la personne $(\mathrm{F}$ ou $\mathrm{H})$, au numéro de codification du questionnaire (de 1 à 164) et à l'âge de l'énonciatrice ou de l'énonciateur. On a aussi indiqué entre crochets, dans le texte même des énoncés, des corrections orthographiques ou de sens, ajoutant les accords ou signalant les fautes de forme et les anglicismes lorsque cela s'imposait.

3. Pour une analyse de certains des thèmes récurrents des revendications des féministes des années 1970, voir l'article de Françoise Picq (1997), tout particulièrement les pages 219-224 ainsi que les premiers chapitres de son livre (1993) et la thèse de Bernier (1991, vol. I). 